\title{
Recent patents in arthritis research
}

\begin{tabular}{|c|c|c|c|c|c|}
\hline Patent \# & Subject & Assignee & Author & Date & Status \\
\hline DE 19809978 & $\begin{array}{l}\text { Soluble member of tumor necrosis factor receptor family with } \\
\text { antiarthritic, antitumor, and antiasthmatic activity. }\end{array}$ & $\begin{array}{l}\text { BASF (Ludwig- } \\
\text { shafen, Germany) }\end{array}$ & Kroeger B & 9/16/1999 & $\mathrm{A} 1$ \\
\hline US 5952197 & $\begin{array}{l}\text { Isolated nucleic acids encoding chemotactic cytokine III; useful } \\
\text { for the prevention, diagnosis, and treatment of leukemia, } \\
\text { inflammatory and autoimmune diseases, psoriasis, and for } \\
\text { promoting wound healing. }\end{array}$ & $\begin{array}{l}\text { Human Genome } \\
\text { Sciences } \\
\text { (Rockville, MD) }\end{array}$ & $\begin{array}{l}\text { Dillon PJ, } \\
\text { Gentz R, Ni J, } \\
\text { Su J, Yu G }\end{array}$ & 9/14/1999 & A \\
\hline WO 9944989 & $\begin{array}{l}\text { Thioamide-derived matrix metalloproteinase inhibitors for } \\
\text { treating conditions involving tissue breakdown and inflammation, } \\
\text { e.g., rheumatoid arthritis, osteoarthritis, and cancer. }\end{array}$ & $\begin{array}{l}\text { Leo Pharm. } \\
\text { Products (Ballerup, } \\
\text { Denmark) }\end{array}$ & Christensen MK & 9/10/1999 & $\mathrm{A} 1$ \\
\hline WO 9944640 & $\begin{array}{l}\text { Use of a combination of a selective NMDA NR2B antagonist } \\
\text { and a COX-2 inhibitor for treating acute or chronic pain with an } \\
\text { inflammatory component, such as rheumatoid or osteoarthritis. }\end{array}$ & $\begin{array}{l}\text { Merck Sharp } \\
\text { \& Dohme } \\
\text { (Whitehouse } \\
\text { Station, NJ) }\end{array}$ & Boyce S & 9/10/1999 & $\mathrm{A} 1$ \\
\hline WO 9943840 & $\begin{array}{l}\text { A chimeric polynucleotide consisting of a tissue necrosis } \\
\text { factor promoter and an apoptosis-inducing Granzyme B } \\
\text { polynucleotide, for treating inflammatory disorders, } \\
\text { especially rheumatoid arthritis. }\end{array}$ & $\begin{array}{l}\text { Boehringer- } \\
\text { Ingelheim Pharm. } \\
\text { (Ridgefield, CT) }\end{array}$ & $\begin{array}{l}\text { Barton RW, } \\
\text { Marlin SD, } \\
\text { Tatake RJ }\end{array}$ & 9/2/1999 & $\mathrm{A} 1$ \\
\hline WO 9943834 & $\begin{array}{l}\text { P-selectin ligand fusion proteins used for treating arthritis, } \\
\text { infections, asthma, diabetes, ulcerative colitis, or transplant } \\
\text { rejection. }\end{array}$ & $\begin{array}{l}\text { Genetics Institute } \\
\text { (Cambridge, MA) }\end{array}$ & $\begin{array}{l}\text { Camphausen R, } \\
\text { Chang X, Cumming } \\
\text { D, Davis M, Kumar R, } \\
\text { Larsen GR, Sako DS, } \\
\text { Shaw G, Veldman GM }\end{array}$ & $9 / 2 / 1999$ & $\mathrm{~A} 2$ \\
\hline WO 9943313 & $\begin{array}{l}\text { New } 3 \mathrm{H}-1,2-\text { dithiolo }(4,3-d) \text { pyrimidine-5,7-dione derivatives } \\
\text { and analogs for use as anticancer, antiproliferative, and } \\
\text { antiinflammatory agents. }\end{array}$ & $\begin{array}{l}\text { Therex Technologies } \\
\text { (Amherst, NY); } \\
\text { State Univ. of NY } \\
\text { Research Found. } \\
\text { (Albany, NY) }\end{array}$ & $\begin{array}{l}\text { Bardos TJ, Dunn JA, } \\
\text { Kung HF }\end{array}$ & 9/2/1999 & $\mathrm{A} 1$ \\
\hline WO 9942436 & $\begin{array}{l}\text { Matrix metalloproteinase-inhibiting } N \text {-hydroxyamide derivatives } \\
\text { used for treating inflammatory diseases including osteoarthritis, } \\
\text { rheumatoid arthritis, and septic arthritis. }\end{array}$ & $\begin{array}{l}\text { American Cyanamid } \\
\text { (Wayne, NJ) }\end{array}$ & $\begin{array}{l}\text { Baker JL, Davis JM, } \\
\text { Grosu GT, Levin JI, } \\
\text { Venkatesan AM }\end{array}$ & 8/26/1999 & $\mathrm{A} 1$ \\
\hline WO 9942101 & $\begin{array}{l}\text { The co-administration of an arachidonic acid metabolism } \\
\text { inhibitor and gamma-linolenic acid to treat inflammatory } \\
\text { diseases, including arthritis. }\end{array}$ & $\begin{array}{l}\text { Wake Forest Univ. } \\
\text { (Winston-Salem, NC) }\end{array}$ & Chilton FH & 8/26/1999 & $\mathrm{A} 1$ \\
\hline WO 9942075 & $\begin{array}{l}\text { An antibody that binds human CD40, for treating } \mathrm{T} \text { cell-mediated } \\
\text { disorders, collagen induced arthritis, and transplant rejection. }\end{array}$ & $\begin{array}{l}\text { Bristol-Myers } \\
\text { Squibb } \\
\text { (Princeton, NJ) }\end{array}$ & $\begin{array}{l}\text { Aruffo AA, Berry KK, } \\
\text { Bajorath J, Harris LJ, } \\
\text { Hollenbaugh D, } \\
\text { Huse WD, Siadak } \\
\text { AW, Thorne BA, } \\
\text { Watkins JD, Wu H }\end{array}$ & 8/26/1999 & $\mathrm{A} 2$ \\
\hline WO 9941382 & $\begin{array}{l}\text { An interleukin-3 polypeptide useful for treating conditions requir- } \\
\text { ing increases in numbers or differentiation of hematopoietic } \\
\text { cells, e.g., immune disorders such as rheumatoid arthritis, or } \\
\text { other conditions in which hematopoietic cell populations have } \\
\text { been reduced/destroyed, e.g., due to radiation or chemotherapy. }\end{array}$ & $\begin{array}{l}\text { Hyseq Inc. } \\
\text { (Sunnyvale, CA) }\end{array}$ & $\begin{array}{l}\text { Crkvenjakov R, } \\
\text { Dickson M, } \\
\text { Drmanac RT, } \\
\text { Drmanac S, Ford J, } \\
\text { Kita D, Labat I, } \\
\text { Leshkowitz D }\end{array}$ & 8/19/1999 & A2 \\
\hline WO 9941369 & $\begin{array}{l}\text { An optimized multicomponent genetic vaccine for treating or } \\
\text { preventing infectious disease, autoimmune diseases, immune } \\
\text { deficiency, asthma, allergy, and cancer. }\end{array}$ & $\begin{array}{l}\text { Maxygen Inc. } \\
\text { (Redwood City, CA) }\end{array}$ & $\begin{array}{l}\text { Howard R, } \\
\text { Punnonen J, } \\
\text { Stemmer WPC, } \\
\text { Whalen RG }\end{array}$ & 8/19/1999 & $\mathrm{A} 2$ \\
\hline WO 9937605 & $\begin{array}{l}\text { New very late antigen- } 4 \text { (VLA-4) antagonists for treating } \\
\text { inflammatory, immune, or autoimmune disorders. }\end{array}$ & $\begin{array}{l}\text { Novartis AG; } \\
\text { Novartis- } \\
\text { Erfindunger } \\
\text { Ver. GmbH (Basel, } \\
\text { Switzerland) }\end{array}$ & $\begin{array}{l}\text { Von Matt PJ, } \\
\text { Wattanasin S }\end{array}$ & 7/29/1999 & $\mathrm{A} 1$ \\
\hline
\end{tabular}

